\section{(2) OPEN ACCESS}

\title{
Newly diagnosed diabetes and outcomes after acute myocardial infarction in young adults
}

\author{
Qinglan Ding (D) , 1,2,3 Erica S Spatz, ${ }^{1,4}$ Kasia J Lipska, ${ }^{1,5}$ Haiqun Lin, ${ }^{1,6}$ John A Spertus, ${ }^{7}$ \\ Rachel P Dreyer, ${ }^{1,8}$ Robin Whittemore, ${ }^{3}$ Marjorie Funk, ${ }^{3}$ Hector Bueno (D) , $, 10,11$ \\ Harlan M Krumholz $z^{1,4,12}$
}

- Additional material is published online only. To view, please visit the journal online (http://dx.doi.org/10.1136/ heartjil-2020-317101).

For numbered affiliations see end of article.

\section{Correspondence to} Dr Harlan M Krumholz, Department of Internal Medicine, Yale School of Medicine, New Haven, Connecticut, USA

harlan.krumholz@yale.edu

Part of this work was presented as a poster presentation at ACC20/WCC scientific session.

Received 10 April 2020 Revised 19 August 2020 Accepted 10 September 2020 Published Online First 20 October 2020
Check for updates

(C) Author(s) (or their employer(s)) 2021. Re-use permitted under CC BY-NC. No commercial re-use. See rights and permissions. Published by BMJ.

To cite: Ding Q, Spatz ES, Lipska KJ, et al. Heart 2021;107:657-666.

\begin{abstract}
Objective To examine prevalence and characteristics of newly diagnosed diabetes (NDD) in younger adults hospitalised with acute myocardial infarction (AMI) and investigate whether NDD is associated with health status and clinical outcomes over 12-month post-AMI.
\end{abstract}

Methods In individuals (18-55 years) admitted with AMI, without established diabetes, we defined NDD as (1) baseline or 1-month $\mathrm{HbA} 1 \mathrm{c} \geq 6.5 \%$; (2) discharge diabetes diagnosis or (3) diabetes medication initiation within 1 month. We compared baseline characteristics of NDD, established diabetes and no diabetes, and their associations with baseline, 1-month and 12-month health status (angina-specific and non-disease specific), mortality and in-hospital complications.

Results Among 3501 patients in Variation in Recovery: Role of Gender on Outcomes of Young AMI Patients study, 14.5\% met NDD criteria. Among 508 patients with NDD, 35 (6.9\%) received discharge diagnosis, 91 $(17.9 \%)$ received discharge diabetes education and 14 $(2.8 \%)$ initiated pharmacological treatment within 1 month. NDD was more common in non-White (OR 1.58, 95\% Cl 1.23 to 2.03), obese (OR $1.72,95 \%$ Cl 1.39 to 2.12 ), financially stressed patients (OR 1.27, 95\% CI 1.02 to 1.58). Compared with established diabetes, NDD was independently associated with better diseasespecific health status and quality of life $(p \leq 0.04)$. No significant differences were found in unadjusted inhospital mortality and complications between NDD and established or no diabetes.

Conclusions NDD was common among adults $\leq 55$ years admitted with $\mathrm{AMI}$ and was more frequent in non-White, obese, financially stressed individuals. Under $20 \%$ of patients with NDD received discharge diagnosis or initiated discharge diabetes education or pharmacological treatment within 1 month post-AMI. NDD was not associated with increased risk of worse short-term health status compared with risk noted for established diabetes.

Trial registration number NCT00597922.

\section{INTRODUCTION}

Diabetes is highly prevalent among individuals hospitalised with acute myocardial infarction (AMI) and is associated with increased risk for cardiovascular complications and short-term and long-term mortality. ${ }^{1-3}$ However, nearly 30\% of patients with AMI over age 55 years have undiagnosed diabetes. ${ }^{4-6}$ In many previous studies, newly diagnosed diabetes (NDD) was linked to worse prognosis after AMI compared with individuals without diabetes, but these results were observed in predominantly older male ( $>55$ years old) populations. $^{7-9}$ Early diagnosis and treatment of diabetes in younger adults can reduce the risk of long-term complications, particularly for ischaemic heart disease. ${ }^{10}$ Adoption of haemoglobin (HbA1c) measurement to complement fasting plasma glucose (FPG) and the oral glucose tolerance test (OGTT) may lead to earlier diagnosis of more patients with diabetes. ${ }^{1112}$ However, the prevalence, characteristics and outcomes of NDD in young adults $(\leq 55$ years) hospitalised with AMI is still unknown.

Because younger patients have a better survival rate and lower incidence of clinical events after $\mathrm{AMI},{ }^{13}$ it is possible elevated $\mathrm{HbA} 1 \mathrm{c}$ in young adults admitted with AMI may not be associated with unfavourable outcomes in the following year, although recognising and treating patients to prevent longterm complications is important. Accordingly, we examined the prevalence and characteristics of young adults (18-55 years) hospitalised with AMI who had NDD, using data from Variation in Recovery: Role of Gender on Outcomes of Young AMI Patients (VIRGO) study.

To better describe current NDD management strategies and explore opportunities for improvement, we also examined the proportion of patients with NDD who received diabetes discharge diagnosis or treatment within 1 month after AMI. We then identified patient characteristics independently associated with NDD in the setting of AMI. Finally, we determined whether patients with NDD had different outcomes compared with other young patients with AMI, with attention to diseasespecific (angina symptoms, physical limitations due to angina, angina-specific quality of life) and nondisease-specific health status outcomes (physical/ mental functioning and overall health) and clinical outcomes (mortality and in-hospital medical complications), during the first 12 months after AMI. Measuring these outcomes changes over time may help determine the prognostic significance of NDD on ischaemic outcomes after AMI in younger patients.

\section{METHODS \\ Participants and study design}

A detailed description of the VIRGO study design, inclusion and exclusion criteria, and interviewing 
procedures has been published previously. ${ }^{14}$ Briefly, VIRGO was a prospective, observational study designed to evaluate factors associated with worse outcomes among young men and women with AMI. A 2:1 ratio of women to men was used to increase the proportion of young women. A total of 3572 participants aged 18-55 years admitted to 103 US and 24 Spanish hospitals with AMI were enrolled from August 2008 through May 2012. The study included 3501 participants (2349 women, 1152 men), with 2985 participants from the USA and 516 from Spain. All study participants provided written informed consent for participation, baseline and follow-up assessment. Each participating hospital obtained institutional review board approval.

\section{Patient and public involvement}

Patients were involved in the design and conduct of the VIRGO study. We received input from young adults who had suffered AMI to understand their lived experience with AMI and assessed patient burden in completing study self-reported outcomes questionnaires. This information assisted us in designing study materials.

\section{Data collection and variables}

Baseline data on patient characteristics were collected from both medical records and standardised in-person interviews during index AMI admission. Follow-up telephone interviews were performed by trained staff at 1 and 12 months. Further details of patient data collected at baseline are in online supplemental eAppendix 1.

\section{Diabetes status assessment}

Patients with AMI were classified into three groups based on diabetes status: established diabetes, NDD, and no diabetes. Established diabetes was defined as having chart diagnosis of diabetes or glucose-lowering medications use at AMI presentation. NDD was defined as $\mathrm{HbA} 1 \mathrm{c} \geq 6.5 \%$ at baseline or 1 -month follow-up, in absence of established diabetes. Additional NDD cases were also diagnosed at discharge and 1-month follow-up if a patient had no history of diabetes but received (1) discharge diagnosis of diabetes or (2) initiation of glucose-lowering medications within 1-month post-AMI. Individuals treated with metformin monotherapy for polycystic ovary syndrome management, in absence of other criteria for diabetes, were not considered NDD. ${ }^{15}$

\section{Outcomes measures}

Health status outcomes

Primary outcomes were disease-specific and non-disease-specific health status. Disease-specific health status was assessed at baseline, 1 month and 12 months after AMI using the Seattle Angina Questionnaire (SAQ). ${ }^{14}$ SAQ-angina frequency, SAQ-physical limitations and SAQ-quality of life domains were evaluated. ${ }^{16}$ Domain scores ranged from 0 to 100 , with higher scores indicating better disease-specific health outcomes. SAQ has been validated by psychometric testing and shown reliable in patients with AMI. ${ }^{16}$

Non-disease-specific health status was measured using the 12-item short-form health survey (SF-12) and Euro-Quality of Life Visual Analog Scale (EQ-5D-VAS). ${ }^{14}$ The instruments' scores range from 0 to 100 , with higher values being more favourable. ${ }^{17}{ }^{18}$ SF-12 assesses mental and physical functioning using mental and physical health composite scales and has documented reliability and validity. ${ }^{17}$ EQ-5D-VAS records participants' selfrated overall health on a $20-\mathrm{cm}$ vertical visual analogue scale, where 0 indicates 'the worse health you can imagine' and 100 indicates 'the best health you can imagine'. ${ }^{18}$ The validity and reliability of EQ-5D has been assessed among individuals with AMI. ${ }^{19}$

\section{Clinical outcomes}

In-hospital mortality and 1-month and 1-year post-AMI mortality were collected by reviewing medical records. In-hospital medical complications during AMI admission, including reinfarction, heart failure, stroke/transient ischaemic attack and haemorrhagic complications were also obtained using chart review.

\section{Statistical analyses}

Descriptive statistics of variables, including baseline characteristics, health status scores and clinical outcomes, stratified according to diabetes status, were presented. Univariate and multivariate logistic regression analyses using a backwardelimination approach were performed to identify baseline characteristics that were independently associated with NDD among patients without established diabetes. We used univariate logistic regression analysis to evaluate differences in baseline characteristics between groups according to their diabetes status. We then combined all variables with a $\mathrm{p}<0.15$ in the univariate analysis into a multivariate model and eliminated variables with the highest $p$ value one at a time until all remaining variables in the model had a two-sided $p<0.05$. We reported ORs and $\mathrm{p}$ values.

A series of linear mixed-effects (LME) regression models, with and without adjustment for baseline covariates, were developed to investigate the association between NDD and the repeated measurements of health status during the 12 months after AMI, using patients with established diabetes as a reference group. A follow-up set of models was fit to compare NDD with those without diabetes. Details of LME models fitting information are provided in online supplemental eAppendix 2.

To investigate the association between mortality and diabetes status, we accommodated binary mortality variable (yes/no) collected longitudinally during hospitalisation, at 1 - and 12 months with mixed-effects logistic regression models, with and without adjustment for baseline covariates. The same fixed effects and random effects were included as those in the LME models.

HbA1c Levels Distributions for Newly Diagnosed Diabetes (NDD) NDD diagnosed by HbA1c $\geq 6.5 \%(n=482)$

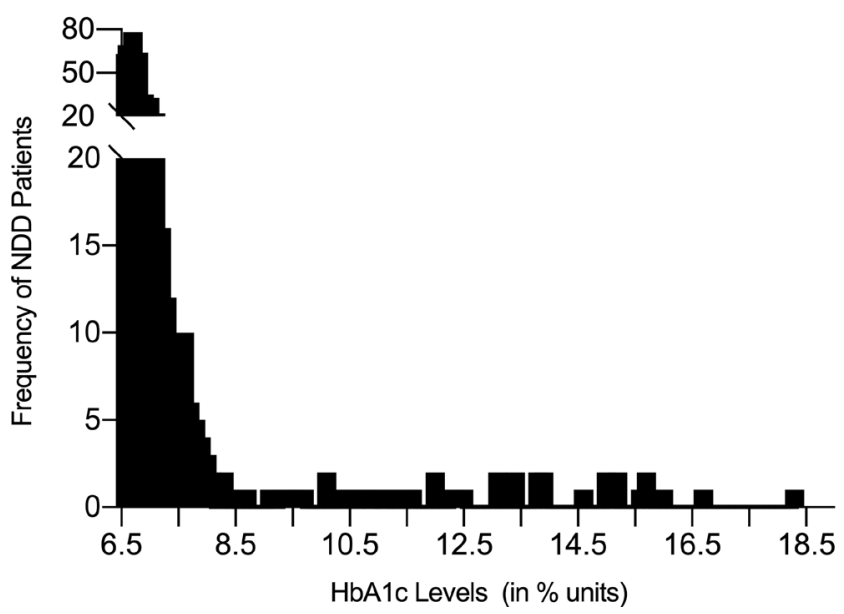

Figure $1 \mathrm{HbA1c}$ levels distribution for newly diagnosed diabetes. 
Table 1 Baseline characteristics of young patients with AMI, stratified by diabetes status

\begin{tabular}{|c|c|c|c|c|c|}
\hline Baseline characteristics & $\begin{array}{l}\text { Without diabetes ( } \mathrm{n}=2008,57.4 \%) \\
\text { (a) }\end{array}$ & $\begin{array}{l}\text { P value* } \\
\text { (b) vs (a) }\end{array}$ & $\begin{array}{l}\text { Newly diagnosed diabetes } \\
(\mathrm{n}=508,14.5 \%)(\mathrm{b})\end{array}$ & $\begin{array}{l}\text { P valuet } \\
\text { (b) vs (c) }\end{array}$ & $\begin{array}{l}\text { Established diabetes }(\mathrm{n}=985,28.1 \%) \\
\text { (c) }\end{array}$ \\
\hline \multicolumn{6}{|l|}{ Sociodemographics } \\
\hline Age in year (SD) & $46.5(6.4)$ & 0.002 & $47.5(5.9)$ & 0.555 & $47.7(5.8)$ \\
\hline Female (\%) & $1270(63.3 \%)$ & 0.954 & $322(63.4 \%)$ & 0.001 & $757(76.9 \%)$ \\
\hline Race & & 0.001 & & 0.188 & \\
\hline White & $1649(82.1 \%)$ & & $381(75.0 \%)$ & & $712(72.3 \%)$ \\
\hline Black & $248(12.4 \%)$ & & $90(17.7 \%)$ & & $212(21.5 \%)$ \\
\hline Others & $111(5.5 \%)$ & & $37(7.3 \%)$ & & $61(6.2 \%)$ \\
\hline Education status & & 0.002 & & 0.057 & \\
\hline Less than high school & $131(6.7 \%)$ & & $13(2.6 \%)$ & & $41(4.2 \%)$ \\
\hline Some high school & $784(39.9 \%)$ & & $202(40.3 \%)$ & & $431(44.4 \%)$ \\
\hline More than high school & $1048(53.4 \%)$ & & $286(57.1 \%)$ & & $498(51.3 \%)$ \\
\hline \multicolumn{6}{|l|}{ Cardiometabolic characteristics } \\
\hline Body mass index $>30 \mathrm{~kg} / \mathrm{m}^{2}$ & $767(38.3 \%)$ & 0.001 & $275(54.2 \%)$ & 0.001 & $667(67.9 \%)$ \\
\hline Peak glucose, median (IQR) & $127.0(38.0)$ & 0.001 & $141.0(48.0)$ & 0.001 & $261.0(162.0)$ \\
\hline Initial systolic blood pressure (mm Hg), median (IQR) & $140.0(37.0)$ & 0.034 & $143.0(38.0)$ & 0.241 & $144.0(42.0)$ \\
\hline Initial diastolic blood pressure (mm Hg), median (IQR) & $87.0(24.0)$ & 0.124 & $88.0(27.0)$ & 0.020 & $86.0(26.0)$ \\
\hline LDL (mmol/L), median (IQR) & $111.0(51.0)$ & 0.729 & $112.0(56.0)$ & 0.008 & $106.0(54.0)$ \\
\hline TG (mmol/L), median (IQR) & $121.5(95.0)$ & 0.001 & $137.0(105.5)$ & 0.001 & $164.0(154.0)$ \\
\hline \multicolumn{6}{|l|}{ CVD risk factors } \\
\hline Family history of CVD & $1395(69.5 \%)$ & 0.034 & $382(75.0 \%)$ & 0.884 & $728(74.1 \%)$ \\
\hline History of hypertension & $1096(54.6 \%)$ & 0.069 & $300(59.1 \%)$ & 0.001 & $821(83.4 \%)$ \\
\hline History of hypercholesterolemia & $1648(82.1 \%)$ & 0.114 & $432(85.0 \%)$ & 0.001 & $922(93.6 \%)$ \\
\hline Smoking within last 30 days & $1241(61.8 \%)$ & 0.878 & $316(62.2 \%)$ & 0.002 & $528(53.7 \%)$ \\
\hline Sleep apnoea & $49(2.5 \%)$ & 0.366 & $16(3.2 \%)$ & 0.001 & $96(9.8 \%)$ \\
\hline \multicolumn{6}{|l|}{ Other comorbidities } \\
\hline History of renal dysfunction & $143(7.2 \%)$ & 0.004 & $56(11.1 \%)$ & 0.004 & $163(16.6 \%)$ \\
\hline History of heart failure & $30(1.5 \%)$ & 0.027 & $15(2.9 \%)$ & 0.001 & $96(9.8 \%)$ \\
\hline Prior Ml & $242(12.1 \%)$ & 0.240 & $71(13.9 \%)$ & 0.001 & $230(23.4 \%)$ \\
\hline \multicolumn{6}{|l|}{ AMI treatment } \\
\hline Coronary revascularisation (PCI/CABG) & $1595(79.4 \%)$ & 0.003 & $433(85.2 \%)$ & 0.412 & $823(83.6 \%)$ \\
\hline Diagnostic angiography & $1900(94.6 \%)$ & 0.001 & $498(98.0 \%)$ & 0.001 & $914(92.8 \%)$ \\
\hline \multicolumn{6}{|l|}{ Discharge medications } \\
\hline Aspirin at discharge & $1948(98.3 \%)$ & 0.459 & $492(98.8 \%)$ & 0.032 & $934(96.9 \%)$ \\
\hline Statin prescribed & $1827(93.4 \%)$ & 0.054 & $471(95.7 \%)$ & 0.397 & $914(94.7 \%)$ \\
\hline Beta-blocker prescribed & $1779(95.1 \%)$ & 0.042 & $459(97.3 \%)$ & 0.506 & $905(36.6 \%)$ \\
\hline ACE or ARB prescribed & $1193(65.8 \%)$ & 0.021 & $329(71.5 \%)$ & 0.005 & $709(78.3 \%)$ \\
\hline \multicolumn{6}{|l|}{ Non-pharmacological interventions prescribed at discharge } \\
\hline Diet counselling & $1850(92.1 \%)$ & 0.659 & $471(92.7 \%)$ & 0.524 & $904(91.8 \%)$ \\
\hline Activity guidelines & $1818(90.5 \%)$ & 0.005 & $480(94.5 \%)$ & 0.001 & $876(88.9 \%)$ \\
\hline Outpatient cardiac rehab prescribed & $874(43.5 \%)$ & 0.132 & $240(47.2 \%)$ & 0.035 & $409(41.5 \%)$ \\
\hline Diabetes education & $166(8.3 \%)$ & 0.001 & $91(17.9 \%)$ & 0.001 & $647(65.7 \%)$ \\
\hline Weight management counselling & $753(37.5 \%)$ & 0.713 & $195(38.4 \%)$ & 0.065 & $427(43.4 \%)$ \\
\hline Smoking cessation counselling & $1391(69.3 \%)$ & 0.339 & $363(71.5 \%)$ & 0.001 & $613(62.2 \%)$ \\
\hline Participated in in-patient cardiac rehab & $519(25.9 \%)$ & 0.010 & $160(31.5 \%)$ & 0.162 & $276(28.0 \%)$ \\
\hline \multicolumn{6}{|l|}{ Clinical characteristics of AMI } \\
\hline Coronary occlusion $\geq 50 \%$ (documented by coronary angiography) & & 0.233 & & & 0.013 \\
\hline Yes & $1637(81.5 \%)$ & & $438(86.2 \%)$ & & $852(86.5 \%)$ \\
\hline No & $244(12.2 \%)$ & & $50(9.8 \%)$ & & $56(5.7 \%)$ \\
\hline Unknown & $127(6.3 \%)$ & & $20(4.0 \%)$ & & $77(7.8 \%)$ \\
\hline ST-segment elevation & $1077(53.6 \%)$ & 0.188 & $289(56.9 \%)$ & 0.001 & $445(45.2 \%)$ \\
\hline Initial heart rate, median (IQR) & $78.0(23.0)$ & 0.003 & $81.0(25.0)$ & 0.001 & $88.0(26.0)$ \\
\hline Peak troponin, median (IQR) & $8.1(31.5)$ & 0.549 & $8.6(25.3)$ & 0.001 & $4.9(20.4)$ \\
\hline Ejection fraction $<40 \%$ & $189(9.6 \%)$ & 0.098 & $60(12.2 \%)$ & 0.818 & $119(12.6 \%)$ \\
\hline Time to presentation $>6$ hours & $767(38.3 \%)$ & 0.999 & $193(38.3 \%)$ & 0.001 & $506(51.6 \%)$ \\
\hline \multicolumn{6}{|l|}{ Other clinical characteristics } \\
\hline Baseline admission HbA1c (\%), median (IQR) & $5.6(0.5)$ & 0.001 & $6.3(1.2)$ & 0.001 & $8.5(3.8)$ \\
\hline 1 month HbA1c (\%), median (IQR) & $6.0(0.5)$ & 0.001 & $6.8(0.4)$ & 0.001 & $7.6(2.0)$ \\
\hline \multicolumn{6}{|l|}{ Diabetes types } \\
\hline Type 1 & NA & & NA & & $104(10.6 \%)$ \\
\hline Type 2 & NA & & NA & & $742(75.3 \%)$ \\
\hline Unknown & NA & & NA & & $139(14.1 \%)$ \\
\hline Peak creatinine, median (IQR) & $0.90(0.3)$ & 0.004 & $0.90(0.3)$ & 0.994 & $0.90(0.4)$ \\
\hline \multicolumn{6}{|l|}{ Psychosocial and behavioural characteristics } \\
\hline Social support via ESSI & $26.1(5.3)$ & 0.151 & $25.7(5.4)$ & 0.082 & $25.2(5.7)$ \\
\hline
\end{tabular}






Data are given as mean (SD), median (IQR) or no. (\%).

* Unadjusted $\mathrm{p}$ values were testing for differences in baseline characteristics between patients with AMI with newly diagnosed diabetes and no diabetes.

tUnadjusted $\mathrm{p}$ values were testing for differences in baseline characteristics between patients with AMI with newly diagnosed diabetes and established diabetes.

AMI, acute myocardial infarction; CABG, coronary artery bypass grafting; CVD, cardiovascular disease; ESSI, ENRICHD social support instrument; HbA1c, glycated haemoglobin; LDL, low-density lipoprotein; MI, myocardial infarction; NA, not available; PCI, percutaneous coronary intervention; PHQ-9, Patient Health Questionnaire-9; PSS, Perceived Stress Scale; TG, triglycerides.

We performed additional analysis by NDD subgroups $(\mathrm{HbA} 1 \mathrm{c}<8 \%$ and $\mathrm{HbA} 1 \mathrm{c} \geq 8 \%)$ and sensitivity analyses of the study cohort. Further details of these analyses and information on missing data are in the online supplemental material (online supplemental eAppendix 3, online supplemental tables 1-7).

\section{RESULTS}

\section{Prevalence of newly diagnosed diabetes}

Among 3501 patients with AMI enrolled in VIRGO, 508 (14.5\%) had NDD and 985 (28.1\%) had established diabetes. The remaining 2008 were classified as having no diabetes (online supplemental figure 1). HbA1c values were available for 2756

Table 2 Univariate and multivariate analyses for the association between baseline characteristics and having newly diagnosed diabetes among young patients with AMI without established diabetes

\begin{tabular}{|c|c|c|c|c|}
\hline \multirow[b]{2}{*}{ Baseline characteristics } & \multicolumn{2}{|l|}{ Univariate } & \multicolumn{2}{|l|}{ Multivariate* $^{*}$} \\
\hline & OR with $95 \% \mathrm{Cl}$ & $P$ value & OR with $95 \% \mathrm{Cl}$ & $P$ value \\
\hline \multicolumn{5}{|l|}{ Sociodemographics } \\
\hline Age in years (mean) & 1.03 (1.01 to 1.04$)$ & 0.003 & $1.02(1.00$ to 1.04$)$ & 0.018 \\
\hline Race Others vs White & $1.53(1.22$ to 1.93$)$ & 0.001 & 1.58 (1.23 to 2.03$)$ & 0.0003 \\
\hline \multicolumn{5}{|l|}{ Cardiometabolic risks } \\
\hline Obesity yes vs no & 1.91 (1.57 to 2.33$)$ & $<0.0001$ & 1.72 (1.39 to 2.12 ) & $<0.0001$ \\
\hline Initial glucose, median (IQR) & $1.01(1.01$ to 1.01$)$ & $<0.0001$ & $1.01(1.00$ to 1.01$)$ & 0.0004 \\
\hline Peak glucose, median (IQR) & $1.01(1.00$ to 1.01$)$ & $<0.0001$ & $1.00(1.00$ to 1.01$)$ & 0.012 \\
\hline Initial systolic blood pressure (mm Hg), median (IQR) & $1.01(1.00$ to 1.01$)$ & 0.004 & Not selected & \\
\hline Initial diastolic blood pressure (mm Hg), median (IQR) & $1.01(1.00$ to 1.01$)$ & 0.006 & Not selected & \\
\hline Triglyceride (mmol/L), median (IQR) & 1.00 (1.00 to 1.002$)$ & 0.002 & Not selected & \\
\hline \multicolumn{5}{|l|}{ CVD risk factors } \\
\hline Family history of CVD & 1.33 (1.06 to 1.66$)$ & 0.012 & 1.30 (1.03 to 1.65$)$ & 0.028 \\
\hline \multicolumn{5}{|l|}{ Other comorbidities } \\
\hline History of renal dysfunction & $1.62(1.17$ to 2.24$)$ & 0.004 & Not selected & \\
\hline History of heart failure & 2.01 (1.07 to 3.76$)$ & 0.029 & Not selected & \\
\hline \multicolumn{5}{|l|}{ AMI treatment during hospitalisation } \\
\hline Diagnostic angiography & 2.83 (1.47 to 5.45$)$ & 0.002 & 2.68 (1.37 to 5.26$)$ & 0.004 \\
\hline Beta-blocker prescribed & $1.83(1.01$ to 3.29$)$ & 0.045 & Not selected & \\
\hline ACE or ARB prescribed & 1.28 (1.03 to 1.59$)$ & 0.027 & Not selected & \\
\hline \multicolumn{5}{|l|}{ Clinical characteristics of AMI } \\
\hline Initial heart rate, median (IQR) & 1.01 (1.00 to 1.01$)$ & 0.009 & Not selected & \\
\hline \multicolumn{5}{|l|}{ Self-reported socioeconomic status } \\
\hline Without health insurance vs with & $1.34(1.06$ to 1.69$)$ & 0.014 & Not selected & \\
\hline Avoided services due to cost (Yes vs no) & $1.50(1.22$ to 1.85$)$ & 0.0001 & 1.27 (1.02 to 1.58$)$ & 0.034 \\
\hline \multicolumn{5}{|c|}{ Have you not taken a medication that your doctor prescribed because of cost? } \\
\hline Always or sometimes (vs rarely to never) & $1.37(1.07$ to 1.75$)$ & 0.013 & Not selected & \\
\hline
\end{tabular}

*The adjusted ORs, $95 \% \mathrm{Cls}$ and p values were estimated from a multiple logistic regression model with the use of backward elimination (All variables left in the final model were significant at the 0.05 level). 
Disease-Specific Health Status (SAQ) Scores Distribution
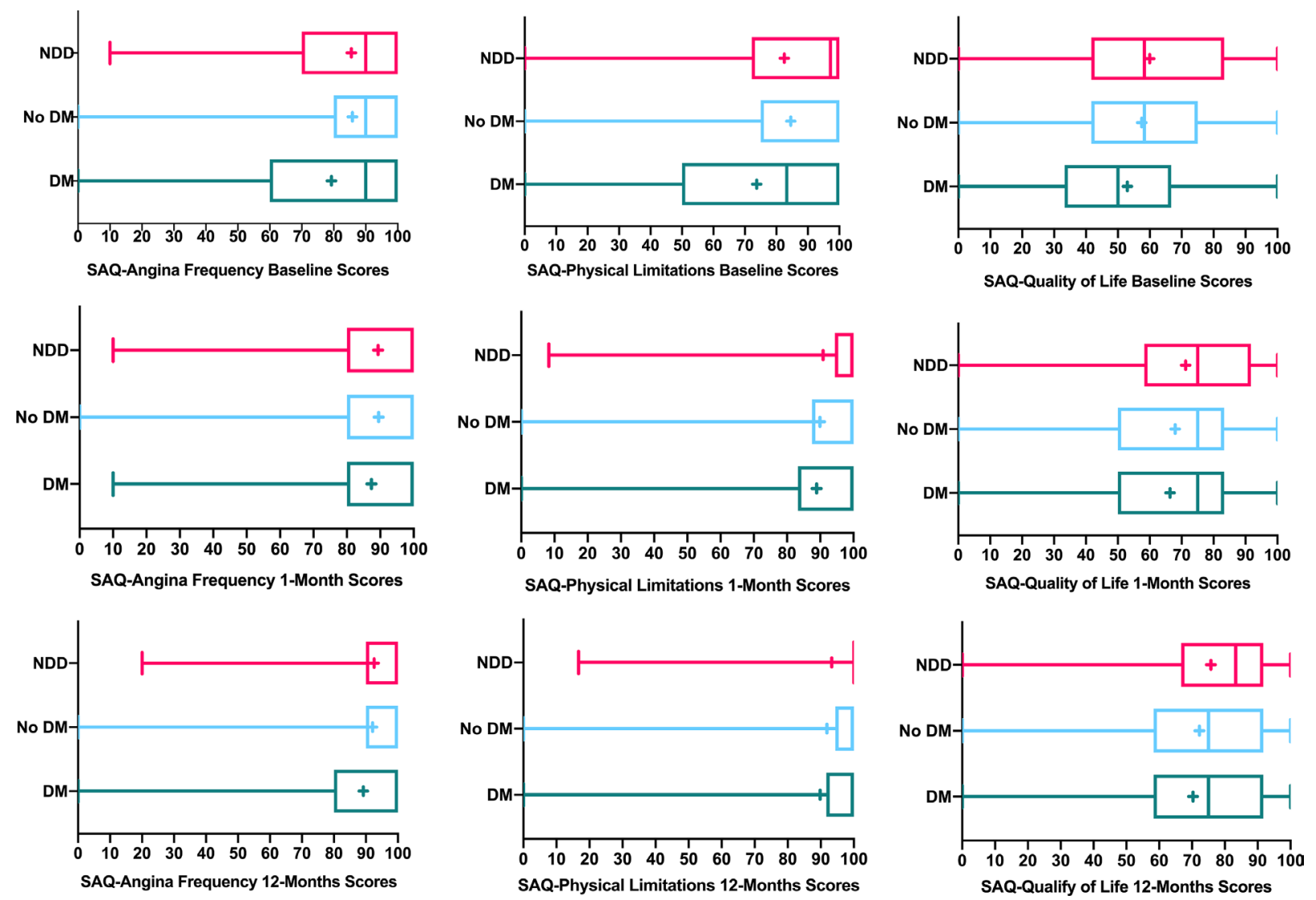

Figure 2 Disease-specific health status scores (SAQ) distribution over time after AMI in young adults, stratified by diabetes status. AMI, acute myocardial infarction; DM, established diabetes; NDD, newly diagnosed diabetes; SAQ, Seattle Angina Questionnaire; box plot showing mean score $(+)$, median score (line within the box), interquartile range box, and the minimum and maximum score values (the ends of the whiskers).

of 3501 patients $(78.7 \%)$ and elevated $(\mathrm{HbA1c} \geq 6.5 \%)$ for 482 patients with NDD (94.9\% of 508 patients with NDD).

Among patients with NDD with elevated HbA1c, the distribution of $\mathrm{HbA} 1 \mathrm{c}$ levels (range $=6.5 \%$ to $18.3 \%$ ) is shown in figure 1 and online supplemental figure 2. Two-thirds had $\mathrm{HbA} 1 \mathrm{c}$ from $6.5 \%$ to $<7.0 \%, 23.4 \%$ had levels of $7.0 \%$ to $<8.0 \%, 1.5 \%$ were between $8.0 \%$ and $9.0 \%$, and $8.3 \%$ were $\geq 9.0 \%$. Of 508 patients with NDD, 35 (6.9\%) had a new diabetes diagnosis at discharge, 91 (17.9\%) received discharge diabetes education (table 1), and diabetes medication was initiated in $14(2.8 \%)$ within 1-month post-AMI.

Sensitivity analysis comparing baseline characteristics between patients with and without missing HbA1c showed those without HbA1c values were significantly younger; had fewer cardiovascular (CVD) risk factors/comorbidities and lower peak glucose levels and were less likely to report barriers to access healthcare at baseline $(\mathrm{p}<0.001)$ (online supplemental table 2).

\section{Patient characteristics}

Baseline characteristics stratified by diabetes status are presented in table 1. Patients with established diabetes and NDD were comparable in age, race and education level, but CVD risk factors of patients with NDD were more similar to patients without diabetes. In these three diabetes status groups, baseline cardiometabolic characteristics and HbA1c levels followed a gradient from lowest (patients without diabetes) to highest (patients with established diabetes). Most aspects of treatment and clinical characteristics of AMI were similar among the three groups. However, patients with NDD were more likely to present with ST-segment elevation myocardial infarction and receive discharge counselling on activity and smoking than patients with established diabetes.

Differences in baseline characteristics between patients with diabetes with and without follow-up are shown in online supplemental table 1 and eAppendix 4. At 12-month follow-up, a significantly higher percentage of patients with NDD reported weight loss after AMI (online supplemental table 7).

Among patients with AMI without established diabetes, the odds of having NDD (versus no diabetes) increased significantly with increasing age or higher initial/peak glucose levels (table 2). Also, NDD was significantly more common in non-White patients (OR 1.58, 95\% CI 1.23 to 2.03 ), those who were obese (OR $1.72,95 \%$ CI 1.39 to 2.12 ), those who had a family history of CVD (OR 1.30, 95\% CI 1.03 to 1.65 ) and those with selfreported avoidance of healthcare due to cost before admission (OR 1.27, 95\% CI 1.02 to 1.58 ).

\section{Longitudinal association between newly diagnosed diabetes and post-AMI health status}

Overall, health status scores improved from baseline to 12 months in all three groups (figures 2 and 3). In the unadjusted analyses, the mean changes of health status scores from baseline to 1 and 12 months were similar between the NDD group and those without diabetes (with the exception of mental functioning) (table 3). For post-AMI angina frequency and disease-specific 
Non-Disease-Specific Health Status (SF-12 \& EQ-5D-VAS) Scores Distribution
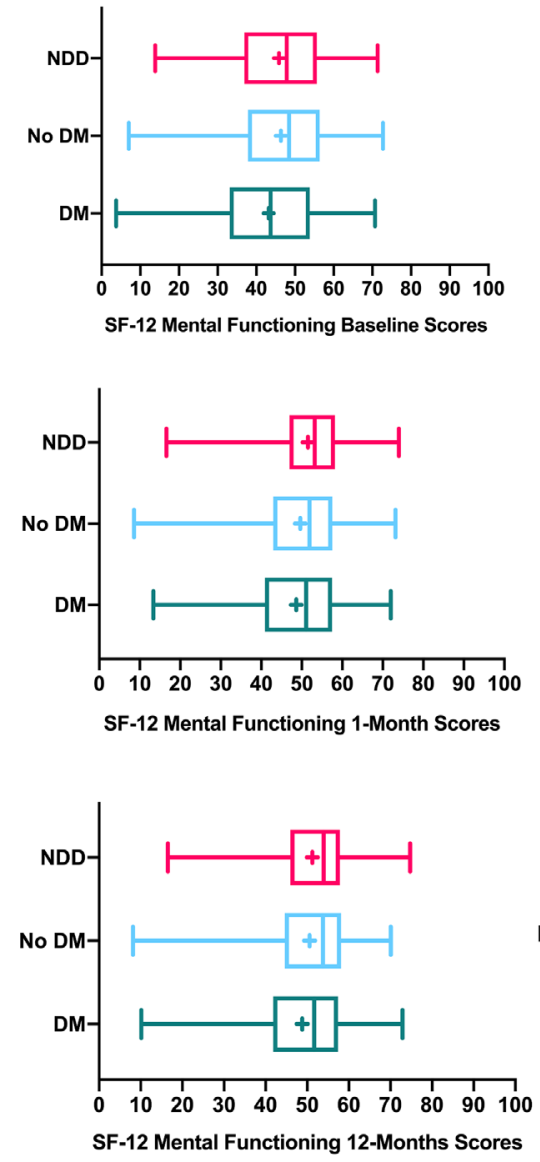
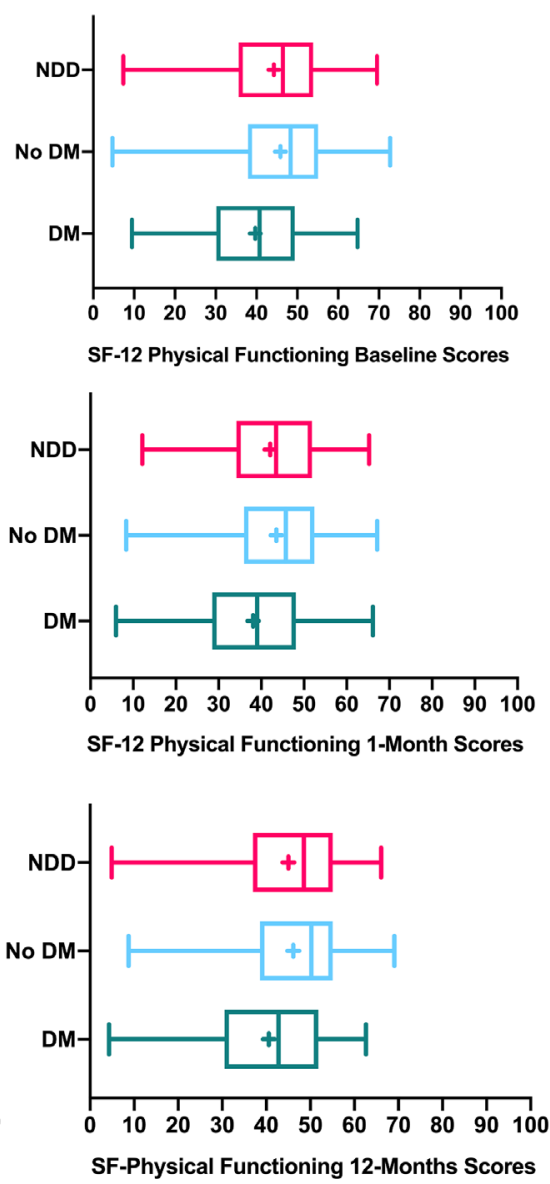
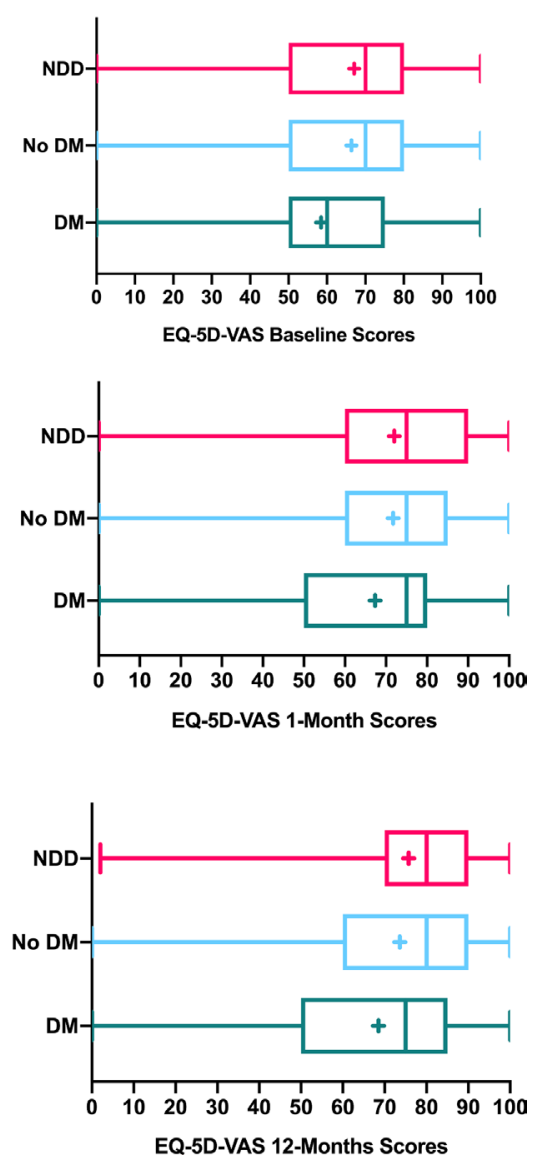

Figure 3 Non-disease-specific health status scores distribution over time after AMI in young adults, stratified by diabetes status. AMI, acute myocardial infarction; DM, established diabetes; NDD, newly diagnosed diabetes; box plot showing mean score (+), median score (line within the box), interquartile range box, and the minimum and maximum score values (the ends of the whiskers).

physical limitations, patients with NDD improved to a lesser extent and more slowly than patients with established diabetes during the 12-month follow-up (tables 3 and 4; $\mathrm{p}<0.03$ for interactions of diabetes status and time, online supplemental figure 3).

We also analysed the independent associations between NDD and health status outcomes in LME models. After adjusting for baseline characteristics, NDD was associated with better disease-specific health status as measured by SAQ, and improved general health status measured by EQ-5D-VAS compared with established diabetes (table 4). In multivariable adjusted subgroup analyses, an HbA1c value of $<8 \%$ in patients with NDD was associated with better disease-specific and general quality of life in young adults with AMI $(\mathrm{p}<0.01)$ (online supplemental table 5). NDD with an HbA1c value $\geq 8 \%$ was associated with increased risk of worse general health status $(p=0.037)$ (online supplemental table 5).

\section{Association between newly diagnosed diabetes and post-AMI clinical outcomes}

The overall mortality rate over the 1-year follow-up for patients with NDD was lowest among the three groups (table 5). Subgroup analyses showed that 1-year mortality was not significantly different between patients with NDD with higher HbA1c $(\geq 8 \%)$ and patients with established diabetes or no diabetes (online supplemental table 4).
Differences between groups for in-hospital medical complications are shown in table 5. Patients with NDD and those without diabetes demonstrated no significant differences in in-hospital complications. Subgroup analysis showed that reinfarction rate during hospitalisation was significantly higher in patients with NDD with higher HbA1c than in those with established diabetes (4.3\% vs $0.9 \%, p=0.033$ ) (online supplemental table 4).

\section{DISCUSSION}

In this multinational cohort of younger adults presenting to hospital with AMI, 14.5\% had NDD. Notably, more than $80 \%$ of individuals with NDD had neither received a discharge diagnosis of diabetes nor initiation of diabetes education or pharmacological treatment within 1-month post-AMI. Thus, the application of a convenient test such as HbA1c in acute settings may help identify more individuals at risk for diabetes complications to facilitate treatment. Compared with patients without diabetes, patients with NDD were more likely to be non-White, obese and financially stressed. Although NDD patients' short-term health status was slightly better than patients with established diabetes, their in-hospital mortality and other complications were not significantly different than others with AMI. The relatively high prevalence of NDD found in this study suggests a clear need to improve detection of risk for diabetes among young patients presenting with AMI. 


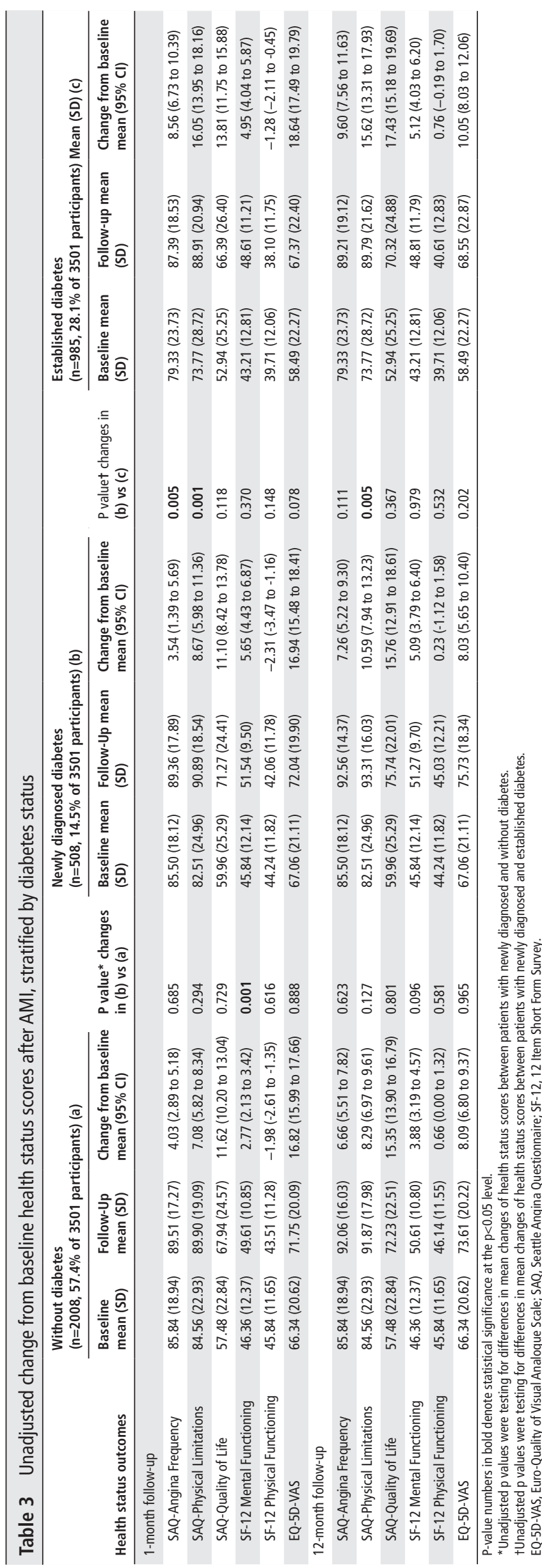

This study extends prior research in several ways. It is the first to examine the prevalence and characteristics of NDD among young people ( $\leq 55$ years) admitted with AMI. Very few studies have evaluated NDD prevalence in this group. Our study, based on a diverse AMI population with a higher proportion of females, supports the relatively high prevalence of NDD in patients with AMI and extends the evidence to younger patients. Despite controversy surrounding the diagnostic role of HbA1c, ${ }^{20}$ elevated $\mathrm{HbA} 1 \mathrm{c}$ was found to be a better predictor of CVD and diabetic retinopathy, compared with FPG or OGTT. ${ }^{21} 22$ Thus, NDD identified by elevated HbA1c and complemented by diabetes discharge diagnosis and treatment may represent a highrisk AMI subgroup and offers a unique opportunity to study the mechanisms of diabetes and micro/macrovascular disease development and potentially to prevent or delay complications.

Second, little data on self-reported socioeconomic status associated with NDD in young AMI populations are available. Sparse research evaluating patient characteristics independently associated with NDD focuses predominantly on clinical characteristics. $^{23}$ Multivariable analysis of patients with AMI in this study took into account financial barriers to healthcare services or medication and identified the independent association of selfreported avoidance of healthcare services due to cost and NDD in a racially diverse young adult population. Because financial barriers to healthcare were a strong predictor of adverse outcomes after $\mathrm{AMI},{ }^{24}$ the avoidance of healthcare services may, in the long-term, have serious undesirable consequences for patients with NDD. These findings support the importance of addressing social determinants in vulnerable populations to promote health. ${ }^{25}$ Further research is needed to understand why a diabetes diagnosis was missed in those with NDD, and why risk factor modification before the events was not carried out effectively.

Last, we demonstrated that NDD is associated with significantly less frequent angina, fewer physical limitations due to angina and better quality of life than established diabetes during a 12-month follow-up. Although somewhat unexpected, these differences may relate to their milder and less symptomatic disease state. It is possible that young adults seeking healthcare leading to an initial diabetes diagnosis tend to do so because they are experiencing symptoms. These findings are supported by evidence suggesting the long asymptomatic period and chronic progression of diabetes. ${ }^{26}$ Additionally, our finding may be partly because we did not adjust for baseline HbA1c since our dataset has a high percentage of missing data on that variable, and we chose not to impute as a reflection of reality. Although research has produced conflicting results on the prognostic importance of HbA1c levels in AMI, some studies have demonstrated that elevated HbA1c was associated with larger infarct size, worse cardiac functioning and long-term mortality. ${ }^{27}$ Our NDD subgroup analysis revealed a trend towards a higher risk of poor health status as HbA1c levels increased. Additional research is needed to confirm the observed trend and understand the impact of baseline HbA1c on post-AMI health status outcomes.

Our findings regarding patients with NDD not having discernably different clinical outcomes than those of patients without diabetes were comparable to research in Korea and China, ${ }^{28}{ }^{29}$ but contradict conclusions from the VALsartan in Acute myocardial iNfarcTion (VALIANT) and Harmonizing Outcomes with RevasculariZatiON and Stents in Acute Myocardial Infarction (HORIZONS-AMI) trials. VALIANT and HORIZONS-AMI trials showed patients with NDD and established diabetes had similarly increased risks of mortality and cardiac events compared with non-diabetics. ${ }^{79}$ Possible reasons for these differences include: 
Table 4 Parameter estimates and P values from the mixed effects models describing the relationship between newly diagnosed diabetes and health status outcomes

\begin{tabular}{|c|c|c|c|c|c|c|}
\hline Health status outcomes & $\begin{array}{l}\text { Estimate (Unadjusted } \\
\text { models) }\end{array}$ & $95 \% \mathrm{Cl}$ & $P$ value & $\begin{array}{l}\text { Estimate (Adjusted } \\
\text { models*) }\end{array}$ & $95 \% \mathrm{Cl}$ & $P$ value \\
\hline \multicolumn{7}{|l|}{ SAQ-Angina Frequency } \\
\hline Newly diagnosed diabetes & 3.79 & 2.29 to 5.31 & $<0.0001$ & 5.05 & 2.50 to 7.59 & 0.0001 \\
\hline \multicolumn{3}{|l|}{ Newly diagnosed diabetes*time2 interaction } & & & & 0.0009 \\
\hline \multicolumn{2}{|l|}{ Newly diagnosed diabetes*time3 interaction } & & & & & 0.0235 \\
\hline Newly diagnosed diabetes & 0.07 & -1.17 to 1.30 & 0.9158 & 0.24 & -0.95 to 1.43 & 0.6928 \\
\hline \multicolumn{3}{|l|}{ Newly diagnosed diabetes*time2 interaction } & & & & 0.8398 \\
\hline \multicolumn{3}{|l|}{ Newly diagnosed diabetes*time3 interaction } & & & & 0.5656 \\
\hline \multicolumn{7}{|l|}{ SAQ-Physical Limitations } \\
\hline Newly diagnosed diabetes & 4.11 & 2.38 to 5.84 & $<0.0001$ & 6.71 & 3.67 to 9.74 & $<0.0001$ \\
\hline \multicolumn{3}{|l|}{ Newly diagnosed diabetes*time2 interaction } & & & & $<0.0001$ \\
\hline \multicolumn{3}{|l|}{ Newly diagnosed diabetes*time3 interaction } & & & & 0.0011 \\
\hline Newly diagnosed diabetes & 0.57 & -0.89 to 2.04 & 0.4450 & -1.55 & -3.7 to 0.60 & 0.1575 \\
\hline \multicolumn{3}{|l|}{ Newly diagnosed diabetes*time2 interaction } & & & & 0.0311 \\
\hline \multicolumn{3}{|l|}{ Newly diagnosed diabetes*time3 interaction } & & & & 0.0183 \\
\hline \multicolumn{7}{|l|}{ SAQ-Quality of Life } \\
\hline Newly diagnosed diabetes & 6.19 & 4.08 to 8.29 & $<0.0001$ & 4.35 & 1.37 to 7.34 & 0.0041 \\
\hline \multicolumn{3}{|l|}{ Newly diagnosed diabetes*time2 interaction } & & & & 0.1143 \\
\hline \multicolumn{3}{|l|}{ Newly diagnosed diabetes*time3 interaction } & & & & 0.2473 \\
\hline Newly diagnosed diabetes Without diabetes & 3.08 & 1.28 to 4.88 & 0.001 & 2.58 & 0.97 to 4.20 & 0.0018 \\
\hline \multicolumn{3}{|l|}{ Newly diagnosed diabetes*time2 interaction } & & & & 0.5455 \\
\hline \multicolumn{3}{|l|}{ Newly diagnosed diabetes*time3 interaction } & & & & 0.5489 \\
\hline \multicolumn{7}{|l|}{ SF-12 Mental Functioning } \\
\hline Newly diagnosed diabetes & 2.82 & 1.83 to 3.81 & $<0.0001$ & 0.33 & -0.88 to 1.56 & 0.5913 \\
\hline \multicolumn{3}{|l|}{ Newly diagnosed diabetes*time2 interaction } & & & & 0.6385 \\
\hline \multicolumn{3}{|l|}{ Newly diagnosed diabetes*time3 interaction } & & & & 0.9505 \\
\hline Newly diagnosed diabetes & 0.96 & 0.07 to 1.85 & 0.0354 & -0.36 & -1.35 to 0.62 & 0.4646 \\
\hline \multicolumn{3}{|l|}{ Newly diagnosed diabetes*time2 interaction } & & & & 0.0005 \\
\hline \multicolumn{3}{|l|}{ Newly diagnosed diabetes*time3 interaction } & & & & 0.1049 \\
\hline \multicolumn{7}{|l|}{ SF-12 Physical Functioning } \\
\hline Newly diagnosed diabetes $\quad$ Established diabetes & 4.31 & 3.19 to 5.43 & $<0.0001$ & 0.77 & -0.40 to 1.94 & 0.1959 \\
\hline \multicolumn{3}{|l|}{ Newly diagnosed diabetes*time2 interaction } & & & & 0.2478 \\
\hline \multicolumn{3}{|l|}{ Newly diagnosed diabetes*time3 interaction } & & & & 0.7762 \\
\hline Newly diagnosed diabetes & -1.43 & -2.38 to -0.48 & 0.0034 & -0.60 & -1.43 to 0.22 & 0.1535 \\
\hline \multicolumn{3}{|l|}{ Newly diagnosed diabetes*time2 interaction } & & & & 0.8236 \\
\hline Newly diagnosed diabetes*time3 interaction & & & & & & 0.6423 \\
\hline EQ-5D Visual Analogue Scale & & & & & & \\
\hline Newly diagnosed diabetes & 6.93 & 5.06 to 8.80 & $<0.0001$ & 4.26 & 1.70 to 6.82 & 0.005 \\
\hline Newly diagnosed diabetes*time2 interaction & & & & & & 0.0036 \\
\hline Newly diagnosed diabetes*time3 interaction & & & & & & 0.3973 \\
\hline Newly diagnosed diabetes & 0.92 & -0.64 to 2.49 & 0.2481 & 1.19 & -0.25 to 2.63 & 0.106 \\
\hline Newly diagnosed diabetes*time2 interaction & & & & & & 0.7887 \\
\hline Newly diagnosed diabetes*time3 interaction & & & & & & 0.2543 \\
\hline
\end{tabular}

$P$ value numbers in bold denote statistical significance at the $p<0.05$ level.

* Models adjusted for cardiometabolic characteristics, gender, sociodemographics, CVD risk factors, other comorbidities, AMI treatment, clinical characteristics of AMI, nonpharmacological interventions, psychosocial and behavioural factors, self-reported socioeconomic status and time.

CVD, cardiovascular disease; EQ-5D-VAS, Euro-Quality of Visual Analogue Scale; NS, not significant; SAQ, Seattle Angina Questionnaire; SF-12, 12 Item Short Form Survey; Time2, indicator of the 1-month follow-up time point; Time3, indicator of the 12-month follow-up time point.

(1) adoption of a healthier lifestyle in NDD group leading to CVD risk optimisation, such as weight loss; (2) VALIANT and HORIZONS-AMI trials did not use HbA1c as one of the criteria to identify patients with NDD and (3) updates of guideline treatment of optimal glycaemic control following AMI. ${ }^{30}$

\section{Study limitations}

Study limitations should be considered in interpreting our findings. First, we did not collect information on diabetes duration and levels of OGTT/ FPG. We also did not use OGTT/ FPG for identifying NDD. Using OGTT/FPG appears to identify different AMI subgroups. ${ }^{27}$ Second, HbA1c was accepted as an additional diagnostic tool for diabetes during the VIRGO study period. ${ }^{11}$ Before 2010, FPG and OGTT were the 'gold standard' for diabetes diagnosis. Although not assessed as part of the current study, differences in the number of NDD cases identified by clinicians using $\mathrm{HbA} 1 \mathrm{c}$ were presumably greater after 2010. Third, we lacked a serial measurement of HbA1c 
Table 5 Mortality and in-hospital medical complications after AMI, stratified by diabetes status

\begin{tabular}{|c|c|c|c|c|c|}
\hline Clinical outcomes & $\begin{array}{l}\text { Without diabetes ( } n=2008 \text {, } \\
57.4 \% \text { of } 3501 \text { participants) } \\
\text { (a) }\end{array}$ & $\begin{array}{l}\text { Newly diagnosed diabetes ( } n=508 \text {, } \\
14.5 \% \text { of } 3501 \text { participants) } \\
\text { (b) }\end{array}$ & $\begin{array}{l}\text { Established diabetes ( } n=985 \text {, } \\
28.1 \% \text { of } 3501 \text { participants) } \\
\text { (c) }\end{array}$ & $\begin{array}{l}\text { P value* } \\
\text { (b) vs (a) }\end{array}$ & $\begin{array}{l}\text { P valuet } \\
\text { (b) vs (c) }\end{array}$ \\
\hline \multicolumn{6}{|l|}{ Mortality } \\
\hline In-hospital mortality & $1(0.1 \%)$ & 0 & $3(0.3 \%)$ & 0.555 & 0.999 \\
\hline 30-day mortality & $12(0.6 \%)$ & 0 & $9(0.9 \%)$ & 0.026 & 0.011 \\
\hline 1-year mortality & $32(1.6 \%)$ & $5(0.9 \%)$ & $35(3.6 \%)$ & 0.0009 & 0.015 \\
\hline \multicolumn{6}{|l|}{ In-hospital medical complications } \\
\hline Re-infarction & $28(1.4 \%)$ & $6(1.2 \%)$ & $9(0.9 \%)$ & 0.403 & 0.927 \\
\hline Heart failure & $109(5.4 \%)$ & $36(7.1 \%)$ & $97(9.9 \%)$ & 0.176 & 0.214 \\
\hline Cardiac arrhythmias & $151(7.5 \%)$ & $32(6.3 \%)$ & $65(6.6 \%)$ & 0.522 & 0.607 \\
\hline Stroke/Transient ischaemic attack & $6(0.3 \%)$ & $2(0.4 \%)$ & $4(0.4 \%)$ & 0.892 & 0.912 \\
\hline Haemorrhagic complications & $153(7.6 \%)$ & $38(7.5 \%)$ & $80(8.1 \%)$ & 0.489 & 0.921 \\
\hline
\end{tabular}

$P$ value numbers in bold denote statistical significance at the $p<0.05$ level.

*Unadjusted $p$ values were testing for clinical outcomes differences between patients with newly diagnosed and no diabetes. Fisher's exact for cells $<5$.

tUnadjusted $\mathrm{p}$ values were testing for clinical outcomes differences between patients with newly diagnosed and established diabetes. Fisher's exact test for cells $<5$.

AMI, acute myocardial infarction.

to confirm a diagnosis of diabetes in all patients. However, our sensitivity analysis supports our assumption that those with missing HbA1c values were less likely to reach the threshold for diagnosis of diabetes. Fourth, a single $\mathrm{HbA1c}$ value limits our ability to explore changes in glycaemic control over time. It is conceivable that some patients with NDD may achieve ideal glycaemic control during follow-up, which could improve their outcomes at 1 year. Finally, we did not adjust for covariates for the comparison of mortality among the three groups due to low

\section{Key messages}

\section{What is already known on this subject?}

- Newly diagnosed diabetes (NDD) is associated with similarly worse prognosis as established diabetes in older adults ( $>55$ years) with acute myocardial infarction (AMI). Little is known about the prevalence and association of NDD with post-AMI outcomes in young adults ( $\leq 55$ years).

\section{What might this study add?}

- Using data from the VIRGO multinational cohort of young adults admitted for AMI, our findings suggest that NDD was frequent among young adults hospitalised with $\mathrm{AMI}$ and was prominent in non-White, obese and financially stressed individuals. Nearly $80 \%$ of patients with NDD had neither received a discharge diabetes diagnosis nor initiation of diabetes education or pharmacological interventions within 1-month post-AMI. NDD was not associated with worse health status compared with risk noted for established diabetes over a 12-month period after AMI. This study suggests that screening to identify NDD in young patients with AMI and improving their cardiometabolic risk profile by pharmacological or lifestyle interventions may help reduce short-term mortality and lead to better health status.

\section{How might this impact on clinical practice?}

- Clinicians should be aware of an increased risk for NDD in young adults hospitalised with AMI, particularly those from disadvantaged backgrounds. The application of a convenient test such as haemoglobin $\mathrm{A} 1 \mathrm{c}(\mathrm{HbA} 1 \mathrm{c})$ in acute settings may help identify more individuals at risk for diabetes complications so that they can be treated. mortality in the NDD group. We also were unable to study the reasons for differences in mortality across these groups. Thus, our findings regarding group differences in unadjusted mortality must be interpreted with caution. Future studies are needed to verify these findings and identify responsible factors.

\section{CONCLUSION}

In this diverse, multinational cohort of young adults hospitalised for AMI, NDD was relatively common and was more prominent in non-White, obese, and financially stressed individuals. Less than $20 \%$ of patients with NDD received a discharge diabetes diagnosis or diabetes education or pharmacological interventions within a month after AMI. Compared with established diabetes, NDD was not associated with an increased risk of worse health status during a 12-month follow-up. These results build on the work of others in support of improved efforts to screen and modify risk factors for diabetes at the time of AMI admission.

\section{Author affiliations}

${ }^{1}$ Center for Outcomes Research and Evaluation, Yale-New Haven Hospital, New Haven, Connecticut, United States

${ }^{2}$ College of Health and Human Sciences, Purdue University, West Lafayette, Indiana, United States

${ }^{3}$ Yale School of Nursing, West Haven, Connecticut, United States

${ }^{4}$ Section of Cardiovascular Medicine, Department of Internal Medicine, Yale School of Medicine, New Haven, Connecticut, United States

${ }^{5}$ Department of Internal Medicine, Section of Endocrinology, Yale School of Medicine, New Haven, Connecticut, United States

${ }^{6}$ School of Nursing, Rutgers University, Newark, New Jersey, United States

${ }^{7}$ Cardiovascular Outcomes Research, St. Luke's Mid America Heart Institute, Kansas City, Missouri, United States

${ }^{8}$ Department of Emergency Medicine, Yale School of Medicine, New Haven Connecticut, United States

${ }^{9}$ Centro Nacional de Investigaciones Cardiovasculares (CNIC), Madrid, Spain

${ }^{10}$ Instituto de Investigacion $i+12$ and Cardiology Department, Hospital Universitario

12 de Octubre, Madrid, Spain

${ }^{11}$ Facultad de Medicina, Universidad Complutense de Madrid, Madrid, Spain

${ }^{12}$ Department of Health Policy and Management, Yale School of Public Health, New Haven, Connecticut, United States

Acknowledgements We appreciate the multiple contributions made by project teams at the Yale-New Haven Hospital Center for Outcomes Research and Evaluation (CORE) in the areas of project operations.

Contributors QD, ES, KJL and HK designed the study and prepared the manuscript for publication. QD and $\mathrm{HL}$ analysed and interpreted the data. All authors contributed to critical revisions of the manuscript. QD and HK are the guarantors of the manuscript. 
Funding The VIRGO study (NCT00597922) was funded by the National Heart, Lung, and Blood Institute (R01 HL081153). IMJOVEN (the Spanish counterpart of the VIRGO study) was supported in Spain by grant PI 081614 from the Fondo de Investigaciones Sanitarias del Instituto Carlos III, Ministry of Science and Technology, and additional funds from the Centro Nacional de Investigaciones Cardiovasculares.

Competing interests $\mathrm{HK}, \mathrm{ES}$ and KJL work under contract with the Centers for Medicare \& Medicaid Services to develop publicly reported quality measures. In the past three years, HK received expenses and/or personal fees from UnitedHealth, IBM Watson Health, Element Science, Aetna, Facebook, the Siegfried and Jensen Law Firm, Arnold and Porter Law Firm, Martin/Baughman Law Firm, F-Prime, and the National Center for Cardiovascular Diseases in Beijing. He is an owner of Refactor Health and Hugo Health, and had grants and/or contracts from the Centers for Medicare \& Medicaid Services, Medtronic, the U.S. Food and Drug Administration, Johnson \& Johnson, and the Shenzhen Center for Health Information. HB receives research funding from the Instituto de Salud Carlos III, Spain (PIE16/00021 \& PI17/01799), Astra-Zeneca, BMS, Janssen and Novartis; has received consulting fees from Astra-Zeneca, Bayer, BMS-Pfizer, Novartis and speaking fees or support for attending scientific meetings from Astra-Zeneca, Bayer, BMS-Pfizer, Novartis and MEDSCAPE-the heart.org. JAS is supported by grants from Bayer, serves as a consultant for Janssen, Bayer, Novartis, AstraZeneca and holds the copyright for the Seattle Angina Questionnaire with royalties paid. KJL is also supported by the National Institute on Aging and the American Federation of Aging Research through the Paul Beeson Career Development Award (K23AG04835).

Patient consent for publication Not required.

Provenance and peer review Not commissioned; externally peer reviewed.

Data availability statement Data are available on reasonable request. The data and study materials will be made available to other researchers if they provide funding support for the deidentification of protected health information in the study and assurances not to share the database on their own.

Supplemental material This content has been supplied by the author(s). It has not been vetted by BMJ Publishing Group Limited (BMJ) and may not have been peer-reviewed. Any opinions or recommendations discussed are solely those of the author(s) and are not endorsed by BMJ. BMJ disclaims all liability and responsibility arising from any reliance placed on the content. Where the content includes any translated material, BMJ does not warrant the accuracy and reliability of the translations (including but not limited to local regulations, clinical guidelines, terminology, drug names and drug dosages), and is not responsible for any error and/or omissions arising from translation and adaptation or otherwise.

Open access This is an open access article distributed in accordance with the Creative Commons Attribution Non Commercial (CC BY-NC 4.0) license, which permits others to distribute, remix, adapt, build upon this work non-commercially, and license their derivative works on different terms, provided the original work is properly cited, appropriate credit is given, any changes made indicated, and the use is non-commercial. See: http://creativecommons.org/licenses/by-nc/4.0/.

\section{ORCID iDs}

Qinglan Ding http://orcid.org/0000-0001-7431-0288

Hector Bueno http://orcid.org/0000-0003-0277-7596

\section{REFERENCES}

1 Ding Q, Spatz E, Lipska K, et al. Screening for newly diagnosed diabetes in young adults hospitalized with acute myocardial infarction-results from the VIRGO (variation in recovery role of gender on outcomes of young acute myocardial infarction patients) study. J Am Coll Cardiol 2020;75:1918.

2 Abbud ZA, Shindler DM, Wilson AC, et al. Effect of diabetes mellitus on short- and long-term mortality rates of patients with acute myocardial infarction: a statewide study. myocardial infarction data acquisition system Study Group. Am Heart J 1995; 130:51-8

3 Vaccarino V, Parsons L, Every NR, et al. Impact of history of diabetes mellitus on hospital mortality in men and women with first acute myocardial infarction. The National Registry of myocardial infarction 2 participants. Am J Cardiol 2000;85:A7:1486-9.

4 Arnold SV, Stolker JM, Lipska KJ, et al. Recognition of incident diabetes mellitus during an acute myocardial infarction. Circ Cardiovasc Qual Outcomes 2015;8:260-7.

5 Giraldez RR, Clare RM, Lopes RD, et al. Prevalence and clinical outcomes of undiagnosed diabetes mellitus and prediabetes among patients with high-risk non-STsegment elevation acute coronary syndrome. Am Heart J 2013;165:918-25.
6 Lankisch M, Füth R, Gülker H, et al. Screening for undiagnosed diabetes in patients with acute myocardial infarction. Clin Res Cardiol 2008;97:753-9.

7 Ertelt K, Brener SJ, Mehran R, et al. Comparison of outcomes and prognosis of patients with versus without newly diagnosed diabetes mellitus after primary percutaneous coronary intervention for ST-elevation myocardial infarction (the HORIZONS-AMI study). Am J Cardiol 2017;119:1917-23.

8 Aggarwal B, Shah G, Randhawa MS, et al. Patients with newly diagnosed diabetes have comparable long term mortality with known diabetics after ST segment elevation myocardial infarction. Circulation 2018;130.

9 Aguilar D, Solomon SD, Køber L, et al. Newly diagnosed and previously known diabetes mellitus and 1-year outcomes of acute myocardial infarction: the valsartan in acute myocardial infarction (VALIANT) trial. Circulation 2004;110:1572-8.

10 Song SH. Complication characteristics between young-onset type 2 versus type 1 diabetes in a UK population. BMJ Open Diabetes Res Care 2015;3:e000044.

11 American Diabetes Association. Diagnosis and classification of diabetes mellitus. Diabetes Care 2010;33 Suppl 1:S62-9.

12 Lugg ST, May CJH, Nightingale P, et al. HbA screening for new onset diabetes following acute coronary syndrome: is it a worthwhile test in clinical practice? J Diabetes Metab Disord 2017;16:14.

13 Rathod KS, Jones DA, Gallagher S, et al. Atypical risk factor profile and excellent long-term outcomes of young patients treated with primary percutaneous coronary intervention for ST-elevation myocardial infarction. Eur Heart J Acute Cardiovasc Care 2016;5:23-32.

14 Lichtman JH, Lorenze NP, D'Onofrio G, et al. Variation in recovery: role of gender on outcomes of young AMI patients (VIRGO) study design. Circ Cardiovasc Qual Outcomes 2010:3:684-93.

15 Barbieri RL. Metformin for the treatment of polycystic ovary syndrome. Obstet Gynecol 2003:101:785-93.

16 Spertus JA, Winder JA, Dewhurst TA, et al. Development and evaluation of the Seattle angina questionnaire: a new functional status measure for coronary artery disease. J Am Coll Cardiol 1995;25:333-41.

17 Ware JE Jr, Kosinski M, Keller SD. A 12-Item short-form health survey: construction of scales and preliminary tests of reliability and validity. Med Care 1996;34:220-33.

18 Rabin R, de Charro F. EQ-5D: a measure of health status from the EuroQol group. Ann Med 2001;33:337-43.

19 Nowels D, McGloin J, Westfall JM, et al. Validation of the EQ-5D quality of life instrument in patients after myocardial infarction. Qual Life Res 2005;14:95-105.

20 Cohen RM, Haggerty S, Herman WH. Hba1C for the diagnosis of diabetes and prediabetes: is it time for a mid-course correction? I Clin Endocrinol Metab 2010;95:5203-6.

21 McCance DR, Hanson RL, Charles MA, et al. Comparison of tests for glycated haemoglobin and fasting and two hour plasma glucose concentrations as diagnostic methods for diabetes. BMJ 1994:308:1323-8.

22 Selvin E, Marinopoulos S, Berkenblit G, et al. Meta-Analysis: glycosylated hemoglobin and cardiovascular disease in diabetes mellitus. Ann Intern Med 2004;141:421-31.

23 Taubert G, Winkelmann BR, Schleiffer T, et al. Prevalence, predictors, and consequences of unrecognized diabetes mellitus in 3266 patients scheduled for coronary angiography. Am Heart J 2003;145:285-91.

24 Rahimi AR, Spertus JA, Reid KJ, et al. Financial barriers to health care and outcomes after acute myocardial infarction. JAMA 2007;297:1063-72.

25 Daniel H, Bornstein SS, Kane GC, et al. Addressing social determinants to improve patient care and promote health equity: an American College of physicians position paper. Ann Intern Med 2018;168:577-8.

26 Harris MI, Klein R, Welborn TA, et al. Onset of NIDDM occurs at least 4-7 yr before clinical diagnosis. Diabetes Care 1992;15:815-9.

27 Timmer JR, Hoekstra M, Nijsten MWN, et al. Prognostic value of admission glycosylated hemoglobin and glucose in nondiabetic patients with ST-segmentelevation myocardial infarction treated with percutaneous coronary intervention. Circulation 2011;124:704-11.

28 Park HW, Kang MG, Kim K, et al. Long-Term prognosis and clinical characteristics of patients with newly diagnosed diabetes mellitus detected after first acute myocardial infarction: from KAMIR-NIH registry. Korean Circ J 2018;48:134-47.

29 Tian L, Wei C, Zhu J, et al. Newly diagnosed and previously known diabetes mellitus and short-term outcomes in patients with acute myocardial infarction. Coron Artery Dis 2013;24:669-75.

30 Kushner FG, Hand M, SmithJr SC, et al. 2009 focused updates: ACC/AHA guidelines for the management of patients with ST-elevation myocardial infarction (updating the 2004 guideline and 2007 focused update) and ACC/AHA/SCAI guidelines on percutaneous coronary intervention (updating the 2005 guideline and 2007 focused update) a report of the American College of cardiology Foundation/American heart association Task force on practice guidelines. J Am Coll Cardiol 2009;54:2205-41. 